\title{
The Influence of Vehicle to Grid Mode on The Economic Benefits of Power Grid
}

\author{
Qian Wei ${ }^{\mathrm{a},{ }^{*}}$, Yuan $A n^{\mathrm{b}}$, Fang $\mathrm{Xi}$, Jiang Yao and Yuyao Wang \\ Institute of water Resources and hydro-electric Engineering, Xi'an University of Science and \\ Technology, Xi'an, 710048, China \\ a402488878@qq.com, b407097615@qq.com
}

\begin{abstract}
Keywords: Electric vehicle; Vehicle to grid; Peak load regulation of power grid; Economic benefit Abstract. With the increasing severity of environmental pollution and fossil resource depletion, electric vehicles draw widespread concern because of their use of clean energy and zero emission. The large-scale popularization of electric vehicles will become an inevitable trend of future development; the two-way interactive technology between vehicle and power grid (Vehicle to Grid, also known as V2G) also receives growing attention. Through V2G technology, power grid's operation inadequacy and inefficiency, limitation on acceptance capacity of renewable energy, inflexible electric vehicle charge and discharge and diseconomy can be relieved to some degree. In this paper, the feasibility of the interaction between the electric vehicle and the power grid was first analyzed, and the composition, function and realization way of the interaction between vehicle and Internet is introduced. Secondly, the Monte Carlo method is used to design a model on electric vehicle's charge and discharge behavior to get the change of daily load curve of the grid after different charge and discharge modes are connected to the grid. Finally, the benefits obtained from the grid in V2G mode are analyzed, and simulation calculation is carried out on electric vehicle's improving effect on load regulation capacity and grid revenue under peak load regulation model.
\end{abstract}

\section{Introduction}

According to relevant forecast, China's electric car ownership will exceed 60 million in 2030 [1]. With the increasing popularity of electric vehicles, interaction between electric vehicles and power grid will become an important part of building a smart power grid. Research shows that more than $90 \%$ electric vehicles' daily average drive time is about 1 hour, and in most of the time they are always in a parking state [2], which in fact represents a kind of idle power resource. If these electric cars were connected to the grid, when the number of cars is enough, they can both charge during valley load and discharge electricity back to the grid during peak load to reduce daily peak-valley load difference, which is namely V2G (Vehicle-to-Grid) concept.

This concept was first proposed by Amory Letendre in 1995. [3] Professor Willett Kempton of the University of Delaware has further developed and studied this concept [4]. Afterwards domestic and foreign scholars began to try to use V2G technology to solve connection problem between power grid and electric car as well as other renewable energy. V2G's feasibility study in China started late compared with foreign countries. However, with the development of electric vehicles in recent years, the research on the two-way interaction between electric vehicles and power grid has gradually increased, including the study of $\mathrm{V} 2 \mathrm{G}$ operation, management and technical problems and solutions [5]. Control and benefit of electric vehicle's large-scale charge and discharge were analyzed: battery loss's impact on V2G benefits and cost of installation were analyzed [6]; potential economic benefits of electric vehicle's involvement in V2G were analyzed, and impact brought by electric vehicle's connection to power grid is discussed [7].

Based on the analysis of the interconnection mode of vehicle and grid, economic benefits of the power grid were analyzed by sampling daily driving laws of electric vehicles when many electric vehicles are connected to gird. 


\section{Composition and function of V2G system}

\subsection{Composition of V2G system}

V2G technology means bidirectional, real-time and controllable power transmission. Bidirectional transmission means that grid can transfer electricity to electric vehicle and in turn electric vehicle can transfer electricity back to the grid. The schematic diagram is as follows

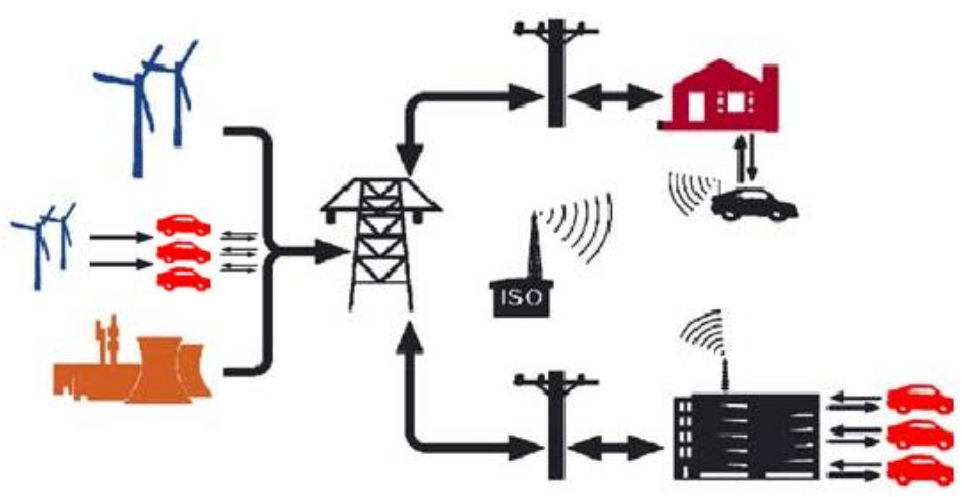

Fig.1. V2G schematic diagram

In the system, the charger can charge electric vehicles in need. Charger's operating voltage, battery's rated capacity and vehicle's charging rate together decide electric car's charging power:

$$
P^{n}(t)=U_{n}(t) \cdot C_{n}(t) \cdot Q_{n}
$$

In this formula, $P^{n}(t)$ _ charging power;

$U_{n}(t)$ charging voltage;

$C_{n}(t)$ _charging rate;

$Q_{n} \_$battery's rated capacity;

In the interconnection (V2G) mode of vehicle-grid, in order to achieve the electric vehicle's charge and discharge function, the V2G charger generally is composited by two-way AC/DC and two-way DC/DC. From the structural point of view, it is actually a cascaded STATCOM, which can achieve reactive power compensation function. Each cascade $\mathrm{H}$-bridge is equipped with a bidirectional DC/DC unit. On the one hand it is designed for electrical isolation. On the other hand because the voltage on DC side of H-bridge is high, reaching up to kilovolt, the electricity cannot be used directly by users and it requires voltage transformation [8].

\subsection{Realization method of V2G}

\subsubsection{Realization method of cluster-type $V 2 G$}

The cluster-type V2G means that to get together all electric vehicles parked in a specific area, which can be an actual parking lot or a virtual aggregator. Most current studies use this approach [9]. As a result of uniform scheduling and centralized management, the overall optimal effect can be achieved. This approach was practiced in this paper.

\subsubsection{Realization method of distributed $V 2 G$}

Electric vehicles involved in autonomous V2G that often scatter everywhere cannot be intensively managed. Therefore, the general practice is using vehicle-mounted smart chargers. They can achieve V2G operation autonomously according to active or reactive power demand and price information released by the grid or according to the electrical characteristics of power grid's outgoing interface and vehicle's own state [10]. 


\section{Modeling of electric vehicle's charge and discharge and their impact on grid's daily load curve}

\subsection{Modeling of electric vehicle's charge and discharge}

The minimum time interval is $5 \mathrm{~min}$, covering 24 hours a day. Electric vehicle's load every $5 \mathrm{~min}$ in a specific area can be expressed as follows:

$$
p_{T i}=\sum_{n=1}^{N} p_{n, i}
$$

$p_{T i}$ is the total load power of electric vehicle at $i^{*} 0.1 h . i=.1,2,3 \ldots 288, \mathrm{~N}$ are the number of electric vehicles in this area. $P_{n, i}$ is the power of No. n electric vehicle at $i * 0.1 h$.

User's behavior is determined by the initial time of battery charge or discharge and the daily mileage of electric vehicle. The time periods that electric vehicles may charge and discharge are 9: 00-17: 30 and 19: 00-7: 00. Charge or discharge's initial time is very close to the normal distribution. The probability density functions of two charge and discharge time periods are as follows:

$$
f_{s 1}(x) \frac{1}{\delta_{s 1} \sqrt{2 \pi}} \exp \left[-\frac{\left(x-\mu_{s 1}\right)^{2}}{2 \delta_{s 1}^{2}}\right]
$$

In this function, $\mu_{s 1}=9, \delta_{s 1}=0.5$.

$$
f_{s 2}(x) \frac{1}{\delta_{s 2} \sqrt{2 \pi}} \exp \left[-\frac{\left(x-\mu_{s 2}\right)^{2}}{2 \delta_{s 2}^{2}}\right]
$$

In this function, $\mu_{s 2}=19, \delta_{s 2}=0.5$.

$$
\begin{gathered}
x \_ \text {mileage of vehicles; } \\
\mu_{s} \_ \text {average value of logarithm; } \\
\delta_{s} \_ \text {variance of logarithm; } \\
f_{s}(x) \_ \text {probability density of mileage. }
\end{gathered}
$$

Daily mileage affects daily power consumption and is related to electric vehicle's battery SOC . The initial SOC of electric vehicle's battery can be expressed as:

$$
\operatorname{SOC}_{0}=\left(1-\frac{a^{*} d}{d_{\max }}\right) * 100 \%
$$

In this formula, $a$ is driving days; $d$ is daily mileage; $d_{\max }$ is the maximum driving distance of electric vehicle.

At a given charge or discharge power, the effective duration is related to the initial battery SOC and the final battery SOC. The charge and discharge duration is as follows: 
Charge:

$$
t_{c}=\frac{\left(s o c_{c}-s o c_{0}\right)}{r_{c i}}
$$

In this formula, $S O C_{c}$ is the $S O C$ of final battery charge. When the battery is fully charged, $S O C_{c}=1 . r_{c i}$ is the charging current. For a battery with a rated capacity of $1 \mathrm{C}$, assuming that the current is $0.1 \mathrm{C}$ and $0.2 \mathrm{C}$, the time needed for battery from empty to fully-charged is $10 \mathrm{~h}$ and $5 \mathrm{~h}$ respectively.

Discharge:

$$
t_{d}=\frac{\left(s o c_{c}-s o c_{d}\right)}{r_{d i}}
$$

In this formula, $S O C_{d}$ is the $S O C$ of final battery discharge. In order to improve battery life, minimum value of $S O C_{d}$ is $0.1 . r_{d i}$ is the discharging current.

\subsection{Charge and discharge behaviors' impact on grid's daily load curve}

(1) Discharge during morning peak:

Set that drive distance in the morning is half of daily mileage. If the battery $S O C>0.5$ after morning drive, the vehicle discharges to the grid. The initial discharge time is 8:30. After this period of discharge, in order to ensure afterward driving, battery SOC should not be less than 0.5.

(2) Discharge during evening peak:

The battery's initial SOC before evening peak discharge is determined by daily driving distance $d$ and early peak discharge power $p_{c}$ which can be achieved by adding consumed power in morning peak and power consumed by the electric vehicle. The initial discharge time is 18:00. Assume that the battery is empty after two discharges. The minimum value of SOC is 0.1 .

(3) After two discharges, owner of electric vehicle charge it at night by connecting to the grid. According to user's behavior, assume that the beginning of charging time is uniformly distributed between 22: 00-24: 00. Electric vehicle's battery starts to charge in the selected charging time until the battery is fully charged. The calculation process of controlled charge and discharge is as follows: 


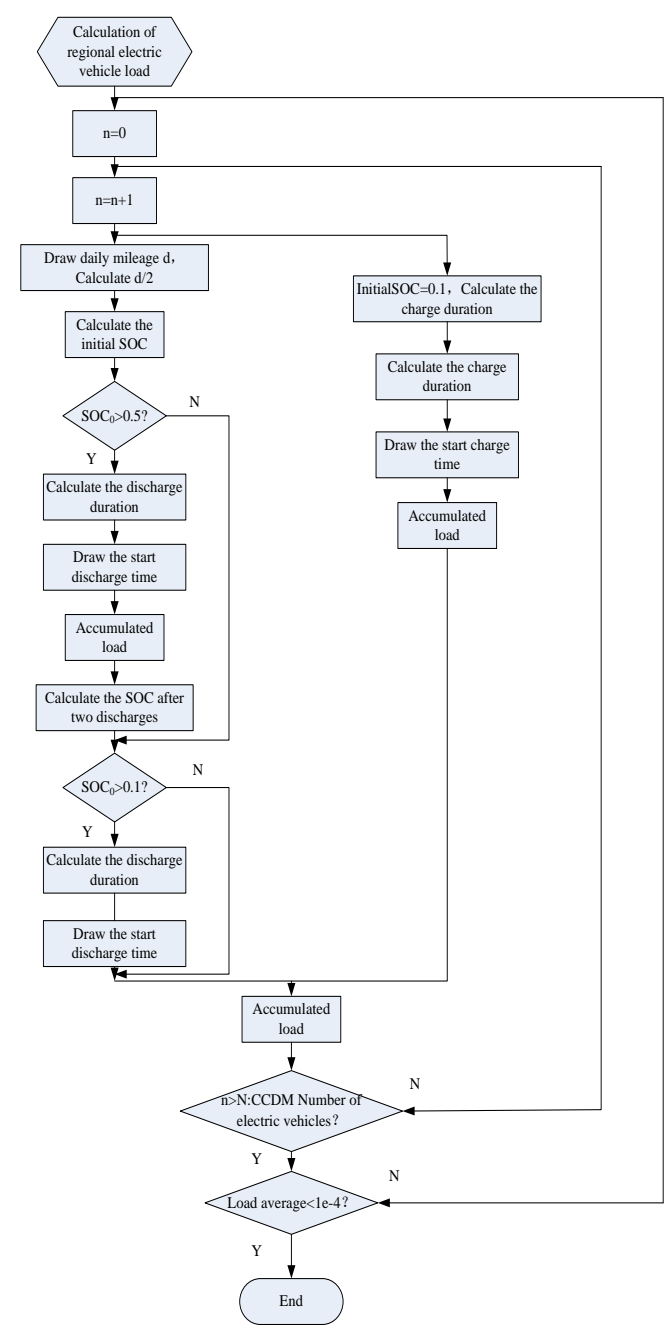

Fig.2. Flow chart of controlled charge and discharge calculation

In accordance with the above controlled charge and discharge strategy, electric vehicle's charging power curve in single V2G mode can be achieved. Assuming that $50 \%$ electric vehicles in this mode is involved in the charge and discharge process, the grid load curve in this mode is obtained by adding the total power of 40,000 electric vehicles and daily load curve. The grid load curve is as follows:

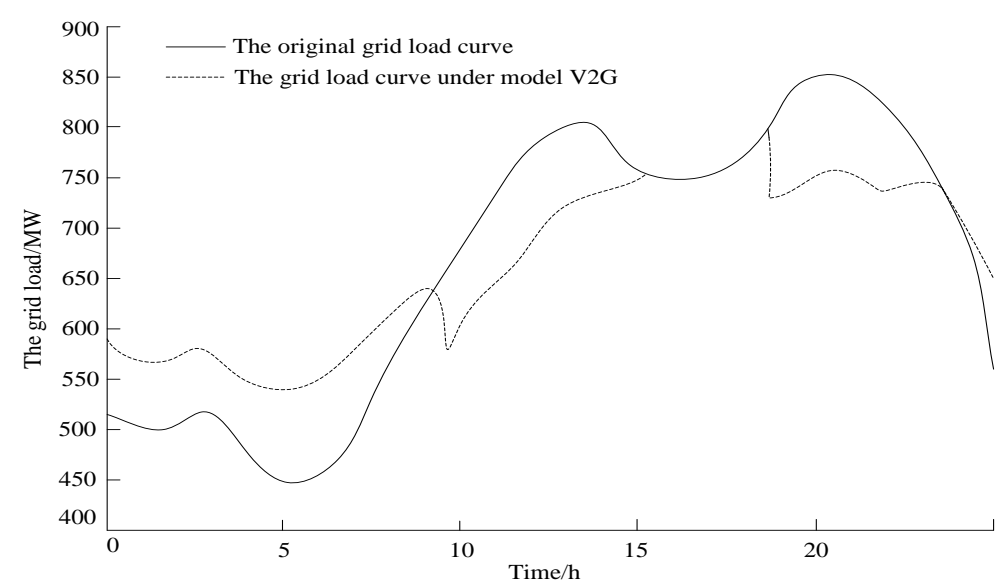

Fig.3.Grid load curve of V2G model

It can be seen from the figure that the two peaks of the power grid are obviously reduce; the grid load of original valley period is also lower than the average load level of the grid; the peak-valley difference is also reduced. This model effectively achieves control purpose of shifting peak load. 
Therefore, the orderly charge and discharge management of electric vehicles is an effective way to shift the peak load.

\section{Economical efficiency study of electric vehicle involved in load regulation of the grid}

\subsection{The process of electric vehicle involved in load regulation}

\subsubsection{Analysis on regional load curve's load regulation strategy}

The grid load curve shown in the following figure is analyzed as an example. The optimal load regulation interval should be between 9:00-12:00. If the user's vehicle can discharge as much as possible in this interval according to its own situation, V2G's load regulation capacity will be optimal. In fact the user may not be able to discharge in this interval because the vehicle's discharge time is also subject to other factors.

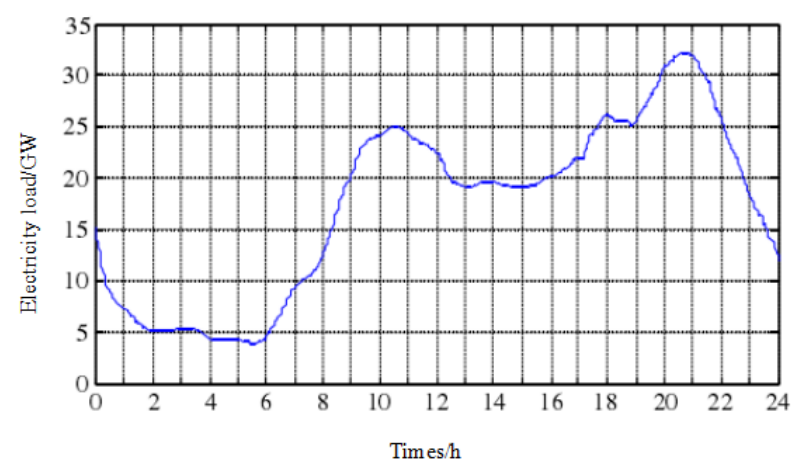

Fig.4.Typical daily load curve of power grid

Electric vehicles involved in load regulation first need to consider the restrictions on electric vehicle's parking interval; the second is restriction on vehicle's remaining SOC and backup SOC.Load regulation's capacity calculation is as follows:

$$
f_{\text {capacity }}=\sum_{i=1}^{N} \sum_{j=1}^{k_{i}} p_{v e h} \eta_{v e h} \Delta t
$$

In this formula: $N \_$total number of electric vehicles parked in charge station every day;

$k_{i} \_$number of discharge period of NO. i vehicle;

$p_{v e h}$ discharge power of electric vehicle;

$\eta_{v e h} \longrightarrow$ discharge efficiency;

$\Delta t$ — time interval between every discharge period.

Restrictions: $\left\{\begin{array}{l}t_{\text {dis_start }} \geq t_{\text {arrive }} \\ t_{\text {dis_start }} \geq t_{\text {peak_on }}\end{array}\right.$ 


$$
\left\{\begin{array}{l}
t_{\text {dis_stop }} \geq t_{\text {leave }} \\
t_{\text {dis_stop }} \geq t_{\text {peak_off }} \\
t_{\text {dis_stop }} \geq t_{\text {soc_off }}
\end{array}\right.
$$

In this formula: $t_{\text {dis_start }}$ _ start time of discharging;

$t_{\text {peak_on }} \longrightarrow$ start time of peak load;

$t_{\text {peak_off }} \longrightarrow$ stop time of peak load;

$t_{\text {soc_off }} \longrightarrow$ stop time of battery capacity limitation;

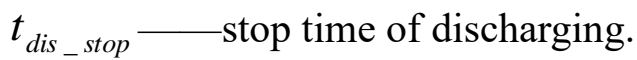

\subsubsection{Analysis on load regulation strategy based on grid price curve}

First, determine the arrival and departure time according to vehicle's parking information, and then determine whether the vehicle can carry out load regulation service according to the vehicle's current battery capacity and backup capacity. Overall revenue formula of charge stations involved in $\mathrm{V} 2 \mathrm{G}$ peak load regulation is as follows:

$$
f_{\text {profit }}=\sum_{i=1}^{N} \sum_{j=1}^{k_{i}}\left(\lambda_{j+t_{\text {arrive }}}-\lambda_{0}-\Delta \lambda\right) p_{v e h} \eta_{v e h \Delta t}
$$

In this formula: $\lambda_{j} \longrightarrow$ electricity price at $\mathrm{j}$ period;

$\lambda_{0} \_$average electricity price during valley load;

$\Delta \lambda \_$charge station's electricity price per capacity.

Restrictions: On period that vehicles can charge: $k_{i} \leq \frac{t_{\text {leave }}^{i}-t_{\text {arrive }}^{i}}{\Delta t}$

On vehicles' SOC: $\operatorname{soc}_{0}^{i} \leq \operatorname{soc}_{\text {reserve }}^{i}$

On charge station's revenue: $\sum_{j=1}^{k_{i}}\left(\lambda_{j+t_{\text {arrive }}}-\lambda_{0}-\Delta \lambda\right) \geq 0$

\subsection{Study on load regulation strategy and simulated analysis}

Simulation parameters are as follows:

(1) Charge station's parking demand is 1000 cars during load regulation period;

(2) After vehicle arrive at the station, remaining SOC is subject to $\mathrm{N}(0.7,0.1)$ normal distribution;

(3) Backup capacity is a certain time of total capacity of daily average mileage. 0.5, 1.0 and 1.5 are selected. 


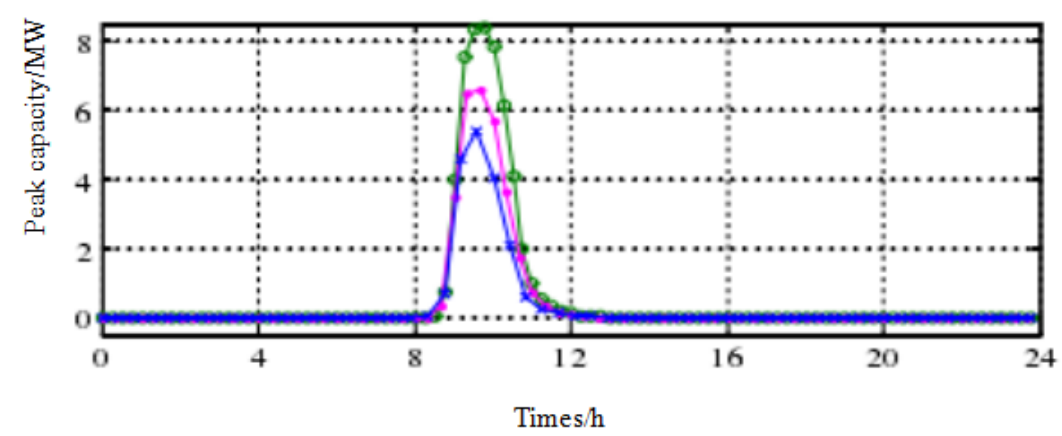

Leave reserve capacity: 0.5 times 1 times

1.5 times

Fig.5. Relationship between peak load shedding and standby capacity based on load curve upsetting

It can be seen from the figure that if peak load regulation period of charge station is 9-12 o'clock, regulation effect is the best, which contributes maximally $10 \mathrm{MW}$ to load regulation capacity. The power of peak load regulation decreases with the increase of backup capacity.

The following figure shows the relationship between the revenue and discharge power of charge station under two load regulation strategies. During calculation process, 0.5 times driving millage capacity is adopted and user's revenue is ignored.

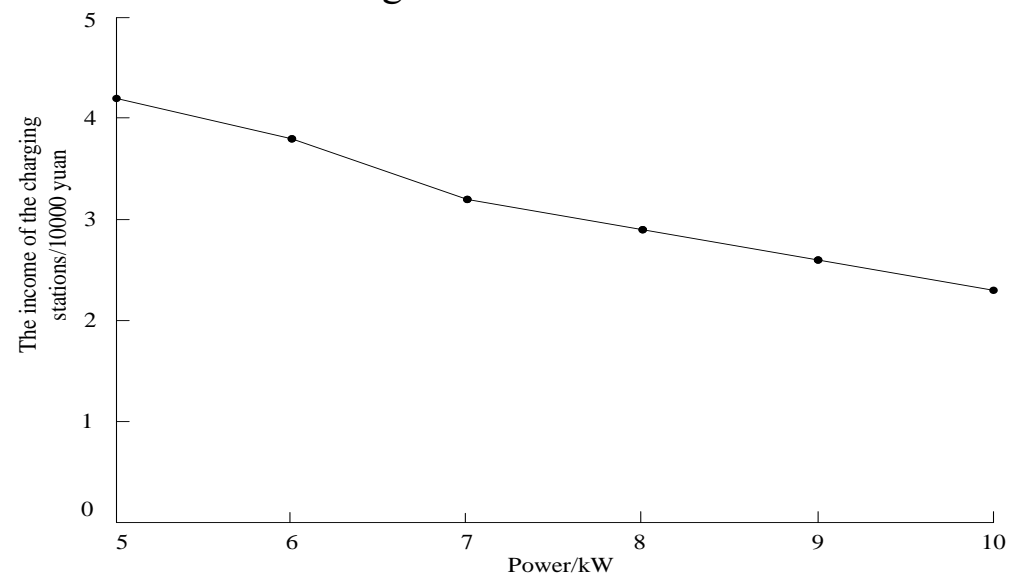

Fig.6.Relationship between charge station's revenue and discharge power

It can be seen from the figure that discharge power has a big influence on V2G charge station's revenue. When regulate the load based on the optimal revenue, the difference of revenue between $5 \mathrm{~kW}$ and $10 \mathrm{~kW}$ discharge power is about $15,000 \mathrm{RMB}$ while based on the optimal regulation period the difference is about 0.5 million.

\section{Summary}

(1) V2G technology is not only conducive to the power grid's economical, reliable operation, but also brings user revenue, which indicates a good prospect.

(2) The Monte Carlo method can be used to sample the driving laws of electric vehicles, which is of great significance to study large-scale connection between electric vehicles and power grid.

(3) Electric vehicle's connection to the grid will influence daily load curve to some degree, which specifically speaking can help regulate load.

(4) Considering the characteristics of grid's daily load in the region, the controlled charge and discharge model as well as regulation strategy based on optimal period are established by combining the electric vehicle's parking demand, usable V2G capacity of electric vehicle and limit on optimal load regulation period. Based on the principle of time-of-use electricity price, the load 
regulation strategy with optimal revenue is given by combining electric vehicle's parking demand and the limitation on usable V2G capacity,.

\section{References}

[1] Liangliang Chen, Hao Zhang, Feng Ni, et al. Discussion on Current Situation and Development of Energy Supply Facilities for Electric Vehicles [J]. Automation of Electric Power Systems, 2011, 35(14):11-17.

[2] Huiling Li, Xiaomin Bai. Electric Vehicle Charging's Influence on Distribution Network and Countermeasures [J]. Automation of Electric Power Systems, 2011(17): 38-43.

[3] Lombardi P, Heuer M, Styczynski Z. Battery switch station as storage system in an autonomous power system: optimization issue[C]//Power and Energy Society General Meeting. Minneapolis, USA: IEEE Communication Society, 2010: 1-6.

[4] Pan F, Bent R, Berscheid A, et al. Locating PHEV exchange stations in V2G[C]//Smart Grid Communications (Smart Grid Comm), 2010 First IEEE International Conference on. IEEE, 2010: 173-178.

[5] Fei Xue, Xianzhang Lei, Yebiao Zhang. A Brand-New Approach of Connecting Electrical Vehicles with Smart Grid from Vehicle-to-Grid Mode to Battery-to-Grid Mode [J]. Power System Technology, 2012, 36 (2): 29-34.

[6] Zhuowei Luo, Zechun Hu, Yonghua Song, et al. Optimized Control of Large-scale Electric Vehicle Charge and Discharge and Revenue Analysis of Capacity [J]. Automation of Electric Power Systems, 2012 (10): 19-26.

[7] Xueyou Chen, Minghao Wen, Wei Chen, et al. A Review of Impact and Economic Benefits of Electric Vehicles Integration into Grid operation [J]. Shaanxi Electric Power, 2013(09): 20-28.

[8] Yingda Zhang, Nian Liu, Jianhua Zhang, et al. Electric Vehicle Exchange Station V2G's Impact on Failure Characteristics of Medium-Voltage Distribution Network [J]. Electric Power Automation Equipment, 2014, 34 (11): 55-61.

[9] Lingling Ma, Jun Yang, Cong Fu, et al. Review on Impact of Electric Vehicle Charging and Discharging on Power Grid [J]. Power System Protection and Control, 2013, (03): 140-148.

[10]Gang Li, Yaozhong Dong, Fushuan Wen, et al. Forecast on Electric Vehicle Charging and Discharging Based on Mobile Social Media [J]. Power System Automation, 2016, 40 (9): 64-7. 